\title{
Membership, binarity, reddening and metallicity of red giant candidates in three southern open clusters
}

\author{
J. J. Clariá ${ }^{1}$, J.-C.Mermilliod ${ }^{2}$, A. E. Piatti ${ }^{3}$ and M. C. Parisi ${ }^{1}$ \\ ${ }^{1}$ Observatorio Astronómico, Universidad Nacional de Córdoba, Argentina \\ email: claria, celeste@oac.uncor.edu \\ ${ }^{2}$ Laboratoire d'Astrophysique de l'École polytechnique fédérale de Lausanne, Switzerland \\ email: Jean-Claude.Mermilliod@epfl.ch \\ ${ }^{3}$ Instituto de Astronomía y Física del Espacio, Bs. As., Argentina \\ email: andres@iafe.uba.ar
}

37 potential members of the red-giant branches of NGC 6192, NGC 6208 and NGC 6268 were observed in the UBV system, while 22 of them were also observed in the DDO system. The observations were made at La Silla and Cerro Tololo Inter-American observatories (Chile), using pulse-counting photoelectric photometers. Coravel radial velocities (RVs) were obtained at La Silla for 24 red giant candidates in the three clusters. Five stars of NGC 6192 have RVs in the narrow range $-8.8 \mathrm{~km} / \mathrm{s}<\mathrm{VR}<-6.4 \mathrm{~km} / \mathrm{s}$, including two new spectroscopic binaries (SBs). Three stars of NGC 6208 - including a new SB - and three stars of NGC 6268 show similar RVs so that their physical membership to NGC 6208 and NGC 6268, respectively, is highly probable. Mean radial velocities are: $-7.7 \pm 0.38 \mathrm{~km} / \mathrm{s}(\mathrm{NGC} 6192),-32.21 \pm 0.28 \mathrm{~km} / \mathrm{s}$ (NGC 6208) and $-15.11 \pm 0.08 \mathrm{~km} / \mathrm{s}$ (NGC 6268). We have also derived the following mean E(B-V) values from Janes (1977) iterative method: $0.63 \pm 0.02$ (NGC 6192), $0.31 \pm 0.02$ (NGC 6208) and $0.43 \pm 0.02$ (NGC 6268).

The colour-magnitude diagram (CMD) of NGC 6192 (Fig. 1, left) was built with the CCD UBV data reported by King (1987). Using $\mathrm{E}(\mathrm{B}-\mathrm{V})=0.63$, the best fit is obtained with the Padova isochrone (Girardi, Bressan, Bertelli, et al. 2000) for $\log \mathrm{t}=8.25(\mathrm{Z}=$ 0.019 ) and $\mathrm{V}-\mathrm{M}_{V}=13.00$. Because there are no modern UBV data available for NGC 6208, we have computed (B-V) indices from the (g1-y) indices of Paunzen \& Maitzen (2001). The number of members appears to be small and the MS fit is not well constrained (Fig. 1, middle). Given $\mathrm{E}(\mathrm{B}-\mathrm{V})=0.32$, one solution results in $\mathrm{V}-\mathrm{M}_{V}=11.15$ and $\log \mathrm{t}=$ $9.10(\mathrm{Z}=0.008)$. Likewise, for NGC 6268 we have computed (B-V) indices from (g1-y) (Fig. 1, right). Using $\mathrm{E}(\mathrm{B}-\mathrm{V})=0.38$, we obtained $\mathrm{V}-\mathrm{M}_{V}=11.25$ and $\log \mathrm{t}=8.35$ for $\mathrm{Z}=$ 0.019 . We have computed for each cluster giant the cyanogen anomaly $\Delta \mathrm{CN}$, defined by Piatti, Clariá \& Minniti (1993). The resulting mean $\Delta \mathrm{CN}$ values for the cluster giants imply: $[\mathrm{Fe} / \mathrm{H}]=+0.29 \pm 0.06$ (NGC 6192), $-0.05 \pm 0.05$ (NGC 6208) and $+0.22 \pm 0.06$ (NGC 6268).

We have found 42 open clusters with known properties whose galactic coordinates differ in $\pm 10^{\circ}$ from those of NGC 6192. Their distribution in the galactic (X,Y) plane is shown in Fig. 2 (left). The galactic center position is given by $(\mathrm{X}, \mathrm{Y}, \mathrm{Z})=(0,0,0)$. NGC 6192 is located behind the Carina spiral arm as seen from the Sun, while NGC6208 and NGC 6268 are placed in front of this feature. Fig. 2 (right) shows the relation between the visual absorption and the distance from the Sun, including that relation for the Baade's window. BH 222 and Westerlund 1 (W1) are the farthest clusters of the selected sample. At the distance of these two clusters, the visual absorption towards Baade's 
window - not far from the direction of NGC 6192 - is between 4 and 8 mags smaller. It is surprising that, in spite of being located at approximately the same distance, these two clusters appear to be affected by such different visual absorptions. The reddening of W1 - the most massive compact young cluster identified in the Local Group up to now (Clark, Neguerla, Crowther, et al. 2005) - is produced in front of the Carina spiral arm, probably caused by a single small dark cloud. Note also that NGC 6216, located farther than $4 \mathrm{kpc}$ from the Sun, is reddened as would be expected if it were situated exactly in the Baade's window direction.
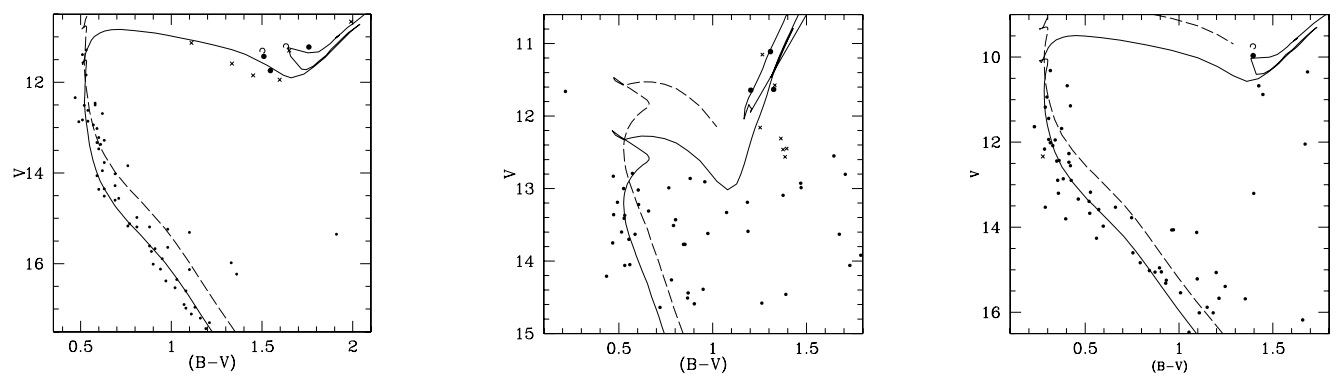

Figure 1. CMDs for NGC 6192 (left), NGC 6208 (middle) and NGC 6268 (right). The Padova isochrones for $\log \mathrm{t}=8.25(\mathrm{Z}=0.019), 9.10(\mathrm{Z}=0.008)$ and $8.35(\mathrm{Z}=0.019)$ have been adjusted to $\mathrm{E}(\mathrm{B}-\mathrm{V})=0.63,0.32$ and 0.38 and $\mathrm{V}-\mathrm{M}_{V}=13.00,11.15$ and 11.25 , respectively. The dashed curves are the same isochrones shifted by 0.75 mag to reproduce the upper binary ridge. For the red giants, filled and open circles stand for single and binary members respectively, while crosses stand for non-members.
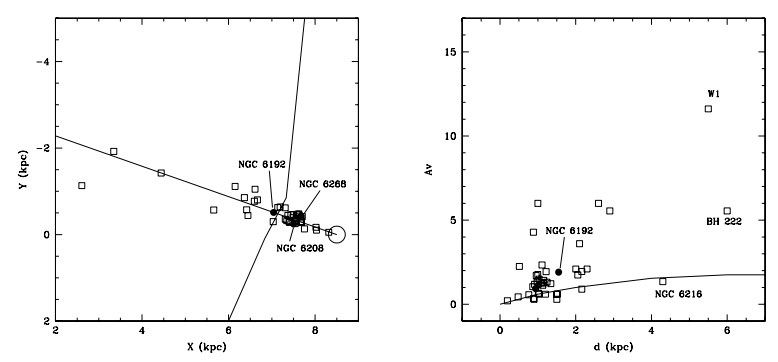

Figure 2. Rectangular (X,Y) coordinates of inner disk open clusters located in the direction of NGC 6192 (left). The Carina spiral arm, the line-of-sight from the Sun to NGC 6192 (straight line) and the position of the Sun are indicated. Visual interstellar absorption vs. distance from the Sun (right). The relationship for the Baade's window is indicated.

Keywords. Galaxy: open clusters, techniques: photometric, radial velocities.

\section{References}

Clark, J.S., Neguerela, I., Crowther, P.A. \& Goodwin, P. 2005, A\&A 434, 949

Girardi, L., Bressan, A., Bertelli, G. \& Chiosi, C. 2000, A\&A 141, 371

Janes, K.A. 2000, PASP 89, 576

King, D.J. 1987, The Observatory 107, 107

Paunzen, E. \& Maitzen, J.M. 2001, A\&A 373, 153

Piatti, A.E., Clariá, J.J. \& Minniti, D. 1993, JA\&A 14, 145 